\title{
A Review of Borderline Personality Disorder
}

\author{
Haoru $\mathrm{Li}^{1, \dagger, *}$ Yubin Tian $^{2, \dagger, *}$ Jiewen Zheng ${ }^{3, \dagger, *}$
}

\author{
${ }^{1}$ Pennsylvania State University, PA, United State \\ ${ }^{2}$ Walt Whitman High School (Maryland), MD, University State \\ ${ }^{3}$ University of Washington, WA, United Stat \\ *Corresponding author.Email: hml5322@psu.edu, shangtiaoshuishu@gmail.com, jiewez@psu.edu \\ These authors contribute equally.
}

\begin{abstract}
This present article reviewed the general situation of borderline personality disorder (BPD), including its definition, prevalence, characteristics, reasons that lead to BPD, and its treatments. The research categorized borderline personality disorder as one of the personality disorders, more specifically, cluster B personality disorder. Three possible reasons and effects for BPD are introduced: childhood experiences, psychological effect, and genetic effect. Several treatments are beneficial to treat patients with BPD, including mentalization-based therapy, dialectical behavior therapy, therapeutic communities, and transference-focused psychotherapy. Each of them is different, but not totally different. Interestingly, they share some of the commonplaces even they are not in the same category. In conclusion, the main causation of borderline personality disorder is the childhood experiences and psychological effects. Also, BPD has more popular-used treatments: mentalization-based therapy and dialectical behavior therapy.
\end{abstract}

Keywords: Borderline Personality Disorder, BPD, review article, treatment

\section{INTRODUCTION}

\subsection{Definition}

Nowadays, despite taking care of physiological health, an increasing amount of people start to pay attention to their psychological health. It is indeed a positive trending that helps more people to adjust their attitudes towards psychological health. While like physiological problems, psychological ones also have plenty of categories on mental disorders. Borderline personality disorder (BPD) is one of the disorders listed under personality disorder according to Diagnostic and Statistical Manual of Mental Disorders fifth edition (DSM-5) criteria [1]. BPD is in cluster B personality disorder, which is characterized by dramatic or unpredictable emotions and actions [2]. People with BPD can undergo the symptoms of unstable emotions and actions, disconnected interpersonal relationship, fear of being abandoned, and difficulty having their self-image [3]. Patients with borderline personality disorder have a great demand for emotional communication with others, and those who communicate with patients need to have relevant experience [4].

\subsection{Patients}

This kind of mental illness can affect family and occupation, as well as personal identity. According to the research on the experience of patients, $71 \%$ of the patients have received physical abuse, and $40 \%$ of them have been abused in childhood [4]. Abuse leads to difficulties in building trusting relationships with others. According to the statistics of hospital patients in the United States, borderline personality disorder patients aged 18-35 accounting for $57.8 \%, 85.5 \%$ of female patients and $82.4 \%$ of Caucasian patients. In the family survey conducted by Patel, most of the patients $(59.2 \%)$ were in middle-class families, $37.8 \%$ had drug abuse and $20.4 \%$ had alcohol abuse. In general, young white women in the United States are more likely to have a borderline personality disorder.

\subsection{Prevalence}

BPD remains prevalent across the psychiatric population (i.e., refers to all individuals who are diagnosed with mental illness), although it might be common in the general population (i.e., refers to all 
individuals without reference of mental illness), thus representing an important public health concern (i.e., a medical issue that affects a significant portion of a specific population) [6]. It tends to become visible during early teenage, worsening as one gets to early adulthood [7]. The prevailing importance of BPD is that the causal to the disease is yet to be established, with various scholars linking it to genetics, environment, social and cultural factors [8]. The research's principal objective is to provide evidence on borderline personality disorder based on its development, particularly using social baseline theory and how it can be treated [6].

\section{CAUSES}

Styles can be applied using the style palette available within the template. To activate it the press Ctrl+Shift+s. Apply the style as required based on the content and context. (Please don't highlight your text in yellow.)

\subsection{Childhood experience}

The study has shown that borderline personality disorder may be predicted by observing a specific childhood state, the main purpose of this study is to compare the characteristics of children with those adults with borderline personality disorder [9]. The same characteristics of the two are impulsive and emotional instability, and so on. According to the research found that children with impulsive childhood, emotional instability, and other characteristics in adulthood have a high risk of suffering from a borderline personality disorder. Childhood conditions can be used to predict adult borderline personality disorder, such as impulsive, emotionally unstable [9]. As for the parents of children with borderline personality disorder, the studies found that most parents are more sensitive than their children [10]. These parents are disgusted that others talk about their children, and even some parents don't like to hear others talk about borderline personality disorder. Parents have a high desire to protect their children [10].

But there are also problems in the above experiment, base on the different age group (the age range of the subjects was 3-23) the characteristics of borderline personality disorder may be different. This is the defect of the test, because there are uncontrollable factors in the tests, leading to the deviation of the prediction. For the explanation, the experimenters believe that some characteristics only exist in childhood and only stable during childhood but disappear in adulthood [9].

Therefore, the characteristics of childhood can only be used as one of the predictive conditions of adult borderline personality disorder, and it cannot be completely concluded that they will suffer from a borderline personality disorder.

\subsection{Psychological Effects}

Several psychological factors can lead to borderline personality disorder. Negative childhood experiences are one of the most common reasons that patients get BPD. Reich et al. had convened 358 patients with BPD to conduct a semistructured research interview for those patients who have BPD, asking questions about their childhood. They were blind to the fact of this research purpose. As a result, there are $91 \%$ of patients with BPD reported that they have experienced physical abuse and $92 \%$ of patients were neglected by their parents before age of eighteen [11]. Moreover, a borderline personality disorder is associated with emotional intelligence. Emotional intelligence is a concept that describes how well people can control their mood. measured by the trait-meta mood scale (TMMS). Patients with BPD usually got less scores on their consciousness of emotion [12].

\subsection{Genetic Effects}

The genetic factors form some basis in understanding how BPD develops. According to Step, first-degree relatives (i.e., siblings, have BPD at ten times more likely to have the disorder). While it is evident the disorder runs across the family no findings are defining the exact amount of the disorder is due to a genetic component. Various twin studies have shown a significant number of the disorder attributed to genetic factors estimated at $42 \%$, with $58 \%$ stemming from other factors. Also, neurological anomalies are found to play an important role, the abnormalities can be traced from the confluence of genetics and past traumatic experiences. Notably, childhood traumatic experiences resulting from a child's brain are markedly delicate and still information tends to contribute significantly to the neural deviation. By having a genetic factor of the disorder, the impact of these environmental factors gets amplified to several degrees.

\section{TREATMENTS}

\section{1. mentalization-based therapy (MBT)}

There are one of the treatments that are correlated with borderline personality disorder: mentalizationbased therapy (MBT) [13]. For MBT, the main part is the process of mentalization since BPD includes traits about the problem of relationship and self-image. Mentalization is the process that we can understand each other and our own according to our subjective state and psychological process [14]. Although there are a lot of psychological disorders that are associated with misleading thoughts (mostly negative), such as major depressive disorder and post-traumatic stress disorder (PTSD), it is still worth noticing that therapist should still focus on the traits for borderline personality disorder 
rather than generally going through the process of mentalization [14]. The primary target for mentalization is to help patients start knowing and getting aware of themselves, including their inner status, emotions, thoughts, and actions.

The therapist's psychotherapeutic attitude should include five characteristics. First is the humility stemming from "do not know". The second is to take the time to identify differences of opinions patiently. Third, accepting different points of view is important. Fourth, ask the patient about his/her experience actively. It is crucial to ask for a detailed description of the experience "WHAT" rather than an explanation of "WHY". Fifth is to carefully avoid the need to understand what doesn't make sense. It is also essential to maintain a great relationship between clinicians and patients. When interpreting the result of mentalization, it is crucial to follow the following six steps. The first is to establish empathy, which is to establish the patient's perspective. This is not the same as agreeing with the patient in any circumstances, but the patient must be shown that the therapist has at least understood his or her perspective. The second step is to explore the event that produced the transference. Actions related to thoughts or emotions require explicit expression and sometimes detail. The third is to accept and understand the formulation of the therapist since it is likely that most of the patient's experience in the transference process is based on reality, even if it is partially connected to reality. The fourth step is to try best to interpret patients. The therapist must imagine sitting side by side with the patient, rather than the opposite. They sit side by side and observe what the patient is thinking and feeling and try to be as curious as possible. The fifth is to get the therapist to come up with diverse points of view, and the last step is to supervise the patient and their own reactions to them [14].

\subsection{Dialectical Behavior Therapy (DBT)}

Generally, Dialectical Behavior Therapy (DBT) represents an approach initially developed by a psychologist, Linehan Marsha, to assist individuals in elevating their cognitive and emotional regulation [15]. It is achieved by familiarizing with triggers that stimulate the reactive states and necessitate identifying and applying better skills to help manage such sequences of feelings, behaviors, and thoughts, hence avoiding harmful reactions. In persons with borderline personality disorder, DBT focuses on involving them in a group or individual therapy basing on the fact that they are emotionally vulnerable [16]. DBT achieves the treatment of such individuals by helping them regulate their emotions, teaching about distress tolerance, and enhancing interpersonal effectiveness and mindfulness.

DBT helps treat borderline personality disorder by helping the affected individuals achieve mindfulness and interpersonal effectiveness by participating in activities such as group works. Mindfulness and interpersonal effectiveness are critical due to their ability to inflict positive behavior change [16]. During the involvement in the respective group discussions, the participating members formulate a topic that greatly moves the affected individual. The individuals can also be encouraged to suggest their topics of interest, which encourages their efficient participation. During such times, they are encouraged to observe and describe their content-wise about the topic, and through this, they develop core interpersonal and mindful skills, which helps manage their moods.

Borderline personality disorder can also be treated through DBT practices that involve distress tolerance and emotion regulation. At this point, the core principle of DBT is to help the patients with the disorder learn how to bear the pain involving unstable mood in a skillful way. The DBT therapist equips the affected individuals with knowledge on how to overcome their condition by teaching survival techniques such as self-soothing, distraction, thinking about the cons and pros of staying in such a condition, and how to improve their moments [17]. Vulnerability to an emotional mind can be reduced through the patients being encouraged to accept that their emotions are acceptable, valid, and real. It helps them realize that other people understand how they feel; hence the acceptance regulates their emotions.

Additionally, DBT can also be essential in minimizing the severity of borderline personality disorder by utilizing mindfulness to achieve emotional regulation simultaneously. During this time, patients are equipped with skills of understanding emotions and identifying their activities, including behaviors, physical sensations, and cognition [15]. Through the process, understanding the emotional dynamics and how they generally occur also equips the patients with skills of evaluating and identifying their emotions. The patient's success in identifying and evaluating their emotions as well is critical since it acts as a precursor to emotional regulation.

\subsection{Schema-Focused Therapy}

Schema-focused therapy refers to a strategy used by health professionals to help people with borderline personality disorder identify the behavior patterns and thoughts responsible for their condition's occurrence. The therapy came into existence in the 1980s after Dr Young realized that cognitive-behavioral therapy, the major therapy used for the treatment of the condition, was turning obsolete on the victims with chronic character logical issues [18]. The whole therapy focuses on meeting the patient's unmet emotional needs during childhood, from which better relationships are learned and nurtured to aid recovery. 
Firstly, the therapy may employ using imagery, which encourages the patients to re-think memories during their early life. It helps equip with knowledge on maladaptive schema development. The borderline patients are induced to reflect the sensations, including sounds and sights, in such memories. They are progressively encouraged to undertake imaginary dialogues with people who appeared around them in such memories and encouraged to plead for the unmet needs. The scenario creates an interconnection with their current feeling, which produces similar sensations, hence improving their mood through the elicited met needs [18].

Secondly, the therapy may take the strategy of soliciting the patients to acquire diaries on any experiences responsible for the initial maladaptive schemas and the corresponding thinking patterns. During the real sessions with the therapists, the patients can relate the earlier Schema's thinking patterns from which they can make a comparison and relate with their associated current behaviors and feelings. The whole activity necessitates satisfying emotional needs that correspond to an individual's mood improvement [18].

Finally, schema therapy can also be used to control and manage individuals with a borderline personality disorder by deploying a chair work strategy for treatment purposes. Chair-work strategy necessitates helping the affected individuals recognize variations occurring in their personality and emotions. During such sessions, the therapist arranges two chairs in an opposite position in which the patients under therapy coordinate their movements between them simultaneously. While in each chair, the patients express varying personality aspects and emotions, both current and past. Additionally, the patients can involve in an imaginative dialogue with their friends, family, or relatives. In such an event, they express their emotional aspects and needs in a single chair and move to the opposite chair and answer themselves as an imaginative individual who can meet such needs. Positivity resulting from the event helps improve emotions and mood [19].

\subsection{Transference-focused Psychotherapy (TFP)}

Transference-focused psychotherapy (TFP) requires the therapist to treat the patient face-to-face (online faceto-face can also work). The therapist guides the patient to find out the problem by communicating with the patient [20]. Transference-focused psychotherapy is a long-term treatment, patients need to meet the therapist at least twice a week, and the treatment time is decided according to the treatment situation. Studies have shown that patients with borderline personality disorder improve a lot through transference-focused psychotherapy [20]. Transference-focused psychotherapy can improve the condition of patients with borderline personality disorder by controlling their emotions. In clinical trials, the antidorsal anterior cingulate cortex (ACC) is considered to regulate the expression of emotion and behavior, and transference-focused psychotherapy can increase the participation of anti-dorsal ACC to achieve the effect of emotion control [21]. In clinical trials, monitoring and recording the data of patients before, during, and after treatment can help to control the patients' emotions. Clinicians analyze the patient's condition through the data and need to pay attention to the impulsive and irritable emotion of the patient [21]. The role of transference-focused psychotherapy is to reduce the impulse of patients, to reduce the symptom of borderline personality disorder.

Transfer-focused psychotherapy can be divided into three parts: early treatment, mid-treatment, and late treatment. In the experiment, according to the treatment status of patients with borderline personality disorder, we found that the effect of transfer-focused psychotherapy on patients with early treatment was more effective than that of patients with late treatment. In clinical trials, by recording the emotional status of patients with borderline personality disorder, it was found that the effect would change in the treatment because of patients' personality and growth environment. Transfer-focused psychotherapy for some patients is not an effective treatment. Clinicians found that changing the period of treatment and conversation skills of transfer-focused psychotherapy can change the effect of the treatment. In general, we can find out the difference through the recorded data of patients with borderline personality disorder and change the method of transfer-focused psychotherapy according to the situation of patients. In this way, transfer-focused psychotherapy will play an effective role in the treatment of borderline personality disorder [22].

\section{CONCLUSION}

As a mental disease, borderline personality disorder has an impact on the life and communication of patients. Impulsivity, irritability, and other emotional problems are the manifestations of borderline personality disorder. The present research has studied the pathogenesis and treatment of borderline personality disorder and found that borderline personality disorder can be formed in childhood, and this kind of mental disease can even be transmitted by the gene. This is an important finding in the understanding of the severity of borderline personality disorder, incorrect treatment can affect patients and future generations. The current studies mainly focus on the treatment of borderline personality disorder, and future research should be devoted to the development of the prevention of borderline personality disorder in childhood. Overall, borderline personality disorder needs more attention from society to reduce social prejudice against mental illness. 


\section{REFERENCES}

[1] American Psychiatric Association. (2013). Diagnostic and statistical manual of mental disorders (DSM-5®). American Psychiatric Pub.

[2]Bender, D. S., Farber, B. A., \& Geller, J. D. (2001). Cluster B personality traits and attachment. Journal of the American Academy of psychoanalysis and Dynamic Psychiatry, 29(4), 551-563.

[3]Leichsenring, F., Leibing, E., Kruse, J., New, A. S., \& Leweke, F. (2011). Borderline personality disorder. The Lancet, 377(9759), 74-84.

[4] Ma, W. F., Shih, F. J., Hsiao, S. M., Shih, S. N., \& Hayter, M. (2009). 'Caring Across Thorns'Different care outcomes for borderline personality disorder patients in Taiwan. Journal of Clinical Nursing, 18(3), 440-450.

[5] Patel, R. S., Manikkara, G., \& Chopra, A. (2019). Bipolar disorder and comorbid borderline personality disorder: patient characteristics and outcomes in US hospitals. Medicina, 55(1), 13.

[6] Gonzalez-Torres, M. A. (2018). Psychodynamic psychotherapies for borderline personality disorders. Current developments and challenges ahead. BJPsych international, 15(1), 12-14.

[7] Stepp, S. D. (2012). Development of borderline personality disorder in adolescence and young adulthood: Introduction to the special section. Journal of abnormal child psychology, 40(1), 1-5.

[8] Kverme, B., Natvik, E., Veseth, M., \& Moltu, C. (2019). Moving toward connectedness-a qualitative study of recovery processes for people with borderline personality disorder. Frontiers in psychology, 10, 430.

[9] Cramer, P. (2016). Childhood precursors of adult borderline personality disorder features: a longitudinal study. The Journal of nervous and mental disease, 204(7), 494-499.

[10] Boucher, M. È., Pugliese, J., Allard-Chapais, C., Lecours, S., Ahoundova, L., Chouinard, R., \& Gaham, S. (2017). Parent-child relationship associated with the development of borderline personality disorder: a systematic review. Personality and mental health, 11(4), 229-255.

[11] Reich, R. B., Vera, S. C., Marino, M. F., Levin, A., Yong, L., \& Frankenburg, F. R. (1997). Reported pathological childhood experiences associated with the development of borderline personality disorder. American Journal of Psychiatry, 154(8), 11011106.

[12] Salovey, P., Mayer, J. D., Goldman, S. L., Turvey, C., \& Palfai, T. P. (1995). Emotional attention, clarity, and repair: Exploring emotional intelligence using the Trait Meta-Mood Scale.

[13] Choi-Kain, L. W., Finch, E. F., Masland, S. R., Jenkins, J. A., \& Unruh, B. T. (2017). What works in the treatment of borderline personality disorder. Current behavioral neuroscience reports, 4(1), 2130 .

[14] Bateman, A., \& Fonagy, P. (2010). Mentalization based treatment for borderline personality disorder. World psychiatry, 9(1), 11.

[15] Bedics, J. (2020). The Handbook of Dialectical Behavior Therapy (1st ed., p. XV-30). London: Academic Press.

[16] Paris, J. (2008). Borderline personality disorder. New York: Guilford Press.

[17] Sperry, L. Handbook of the diagnosis and treatment of DSM-5 personality disorders (3red.). New York: Routledge.

[18] Arntz, A., \& Van Genderen, H. (2009). Schema Therapy for Borderline Personality Disorder (1st ed., pp. 7-91). Chichester: John Wiley \& Sons.

[19] Pugh, M. (2020). Cognitive-behavioral chair work. New York: Routledge.

[20] Kernberg, O. F., Yeomans, F. E., Clarkin, J. F., \& Levy, K. N. (2008). Transference focused psychotherapy: Overview and update. The International Journal of Psychoanalysis, 89(3), 601620.

[21] Perez, D. L., Vago, D. R., Pan, H., Root, J., Tuescher, O., Fuchs, B. H., \& Stern, E. (2016). Frontolimbic neural circuit changes in emotional processing and inhibitory control associated with clinical improvement following transferencefocused psychotherapy in borderline personality disorder. Psychiatry and clinical neurosciences, 70(1), 51-61.

[22] Spinhoven, P., Giesen-Bloo, J., van Dyck, R., Kooiman, K., \& Arntz, A. (2007). The therapeutic alliance in schema-focused therapy and transference-focused psychotherapy for borderline personality disorder. Journal of consulting and clinical psychology, 75(1), 104. 